\title{
Investigation of Sensing Capabilities of Organic Bi-layer Thermistor in Wearable e-Textile and Wireless Sensing Devices
}

\author{
Victor Lebedev ${ }^{1}$, Elena Laukhina ${ }^{1,2, *}$, Vladimir Laukhin ${ }^{1,2,3}$, Andrey Somov ${ }^{4} *$, \\ Alexander Baranov ${ }^{5}$, Concepcio Rovira ${ }^{1,2}$, and Jaume Veciana ${ }^{1,2}$ \\ ${ }^{1}$ Institut de Ciencia de Materials de Barcelona (ICMAB-CSIC), Campus UAB, Bellaterra, 08193, \\ Spain \\ ${ }^{2}$ CIBER de Bioingeniería, Biomateriales y Nanomedicina (CIBER-BBN), Madrid, Spain \\ ${ }^{3}$ Institució Catalana de Recerca i Estudis Avançats (ICREA), Barcelona, Spain \\ ${ }^{4}$ College of Engineering, Mathematics and Physical Sciences - University of Exeter, UK \\ ${ }^{5}$ Moscow Aviation Institute (National Research University), Russia \\ *laukhina@icmab.es
}

\begin{abstract}
This study, being stimulated by the discovery of high sensitivity of nanostructured layers of organic semiconductor $\alpha$ '-BEDT-TTF) $2 \mathrm{I}_{\mathrm{x}} \mathrm{Br}_{3-\mathrm{x}}$ [BEDT-TTF= bis(ethylendithio)tetrathiafulvalene] to heat radiation, is strongly related to the field of Organic electronic. This work presents the development and assessment of flexible lightweight highly sensitive filmbased thermistor as (i) a separate sensor, (ii) sensor integrated in e-textile and (iii) sensor embedded in a wireless sensor node. Wireless Sensor Networks (WSN) ) and Internet of Things (IoT), being two promising technologies, have been already applied in a number of monitoring scenarios. In spite of great progress achieved in sensing technologies and wireless embedded systems there is a gap in multidisciplinary research aimed at investigation of potential of these technlogies at a time. Experimental results demonstrate that the developed a bi-layer organic thermistor has high potential in an environmental and biomedical monitoring. They can be used as a part of wearable units or as sensing units on board of wireless sensing devices.
\end{abstract}

Keywords: e-textile, organic molecular conductors, temperature sensor, wireless sensor network

\section{Introduction}

S.R. Forest and $M$ Thompson indicated conclusively in their well known "Introduction: Organic Electronics and Optoelectronics" that the use of organic compounds as active materials in electronic and optoelectronic devices opens the door to a large number of efficient and potentially low-cost methods for fabricating useful, and, in some cases, complicated structures that are inaccessible by conventional methods using conventional semiconductors [1]. It should be noted that one of the important areas for innovation in the field of organic electronics is sensing [2]. For example, it was shown that some of organic molecular conductors, such as trihalides of bis(ethylenedithio)tetrathiafulvalene (BEDT-TTF), are able to give electronic devices extraordinary strain, pressure and temperature sensing properties impossible to achieve with metal-based electronic structures, enabling a broad range of innovative "out-of-thebox" applications $[3,4,5,6]$. On the other hand, the attraction of (BEDT-TTF) ${ }_{2} \mathrm{Hal}_{3}$ - 
organic molecular conductors is the ability to directly impact the sensing properties of the material when deposited in thin film form $[3,7]$.

As noticed earlier, sensing is an emerging technology which can be significantly upgraded by information and communication technologies which are able to send measured data to the user over the network including the Internet. The term Internet of Things (IoT) was coined by Kevin Ashton in 1999 and was about empowering computers with gathering information by means of sensors and RFID technology [8]. Nowadays, there is no a single definition for IoT, but its common understanding is about the devices, objects and services joined in a global network with some processing and cognitive capabilities [9]. Since the time the IoT was first mentioned, sensing, networking and processing technologies have greatly evolved.

For example, there is a great progress in film based electronic components, e.g. sensors and storage [10], which can be deposited right on the surface of the embedded systems. The reason for this progress is grounded on newly available materials, which can ensure better performance, full customization of printed/deposited devices and, in most cases, a simple fabrication process. However, there is still a gap between the printed devices and their integration with embedded electronics and, in particular, with Wireless Sensor Network (WSN) devices. In fact the WSN paradigm is considered as a pillar technology in the forthcoming era of the Internet of Things (IoT) and was recently applied to a number of monitoring scenarios [11, 12] including the medical ones [13]. The IoT is a promising technology in terms of addressing a number of problems [14] including a remote medical assistance $[15,16]$ which appears to be an important challenge in the growing urban areas.

With the development of WSN and IoT there is a growing interest to the sensing technology on plastic substrates . This technology allows for significant production cost reduction and adding new functionalities [1, 17]. Hence there is a high potential for integration of lightweight temperature sensing materials into human wearable interfaces, e.g. fabrics. Wearable electronics is relevant since it offers personalized healthcare, security and comfort [18].

Covering polymeric films with conventional metals and semiconductors is one of the traditional approaches to building up flexible lightweight sensors [19]. For instance, Chun-Chih Huang et al. [20] proposed a flexible thermistor fabricated by printing a square $\mathrm{NiO}$ thin film on a polyimide film. Such flexible bi-layer (BL) sensors are characterized by good sensitivity and fast response time. However, these flexible sensing materials have a common drawback: poor adhesion of conventional metals, as well as their oxides, to polymers. The disadvantage of this kindleads to a low binding between inorganic sensing layers and plastic supports.

To address this problem, organic conductors can be used. A number of research works reported on the point that conducting polymers can be successfully applied to sensing technology [21]; the demonstrated flexible capacitive-type humidity and temperature sensors - cellulose-polypyrrole nanocomposites - are the promising examples of all-organic flexible sensors [22]. At the same time, electronic properties of conducting polymers are not stable enough since they are sensitive to atmospheric 
moisture [23]. Moreover, high manufacturing costs result in expensive conductive polymers which prevents their usage in real applications.

As mentioned earlier, molecular conductors was proposed recently: (BEDT-TTF) ${ }_{2} \mathrm{X}_{3}$ [BEDT-TTF $=$ Bis(ethylenedithio)tetrathiafulvalene, $\mathrm{X}_{3}=$ trihalide anion]. It can be successfully combined with plastic films by relying on a low cost simple synthetic procedure described in $[24,25]$. This procedure shows that conducting bi-layers polycarbonate/(BEDT-TTF) ${ }_{2} \mathrm{X}_{3}$ are highly effective as inexpensive lightweight strain [3], temperature [4] and humidity sensors [26] in terms of their application to a remote patient monitoring. Another significant advantage is integration of the strain sensing polycarbonate/polycrystalline (001) oriented $\alpha$-(BEDT-TTF $)_{2} \mathrm{I}_{3}$ bi layer film into a polyester textile. Resultatnt conducting textile ensures high flexibility and easy detectable electrical response signal even to small strain [27].

In our recent work, we have reported on the bi layer film: polycarbonate/polycrystalline (001) oriented $\alpha^{\prime}$-(BEDT-TTF $)_{2} \mathrm{I}_{\mathrm{x}} \mathrm{Br}_{3-\mathrm{x}}$ molecular conductor [hereafter it will be as polycarbonate/ $\alpha^{\prime}$-(BEDT-TTF) $)_{2} \mathrm{I}_{\mathrm{x}} \mathrm{Br}_{3-\mathrm{x}}$ ] (Fig. 1) which can be used both as a direct-contact thermometer and as a passive noncooled infrared sensor [4]. This sensor is capable of identifying even negligible temperature changes with accuracy of 0.005 degree [4]. The obtained results improve those obtained from widely used thermistors: the accuracy of a Pt-1000 detector is 0.01 degree [28].

In this work we investigate the sensing capabilities of the polycarbonate $/ \alpha$-(BEDT$\mathrm{TTF})_{2} \mathrm{I}_{\mathrm{x}} \mathrm{Br}_{3-\mathrm{x}}$ bi-layer film with the goal to figure out whether it can be used in wearable biomedical and environmental monitoring scenarios.

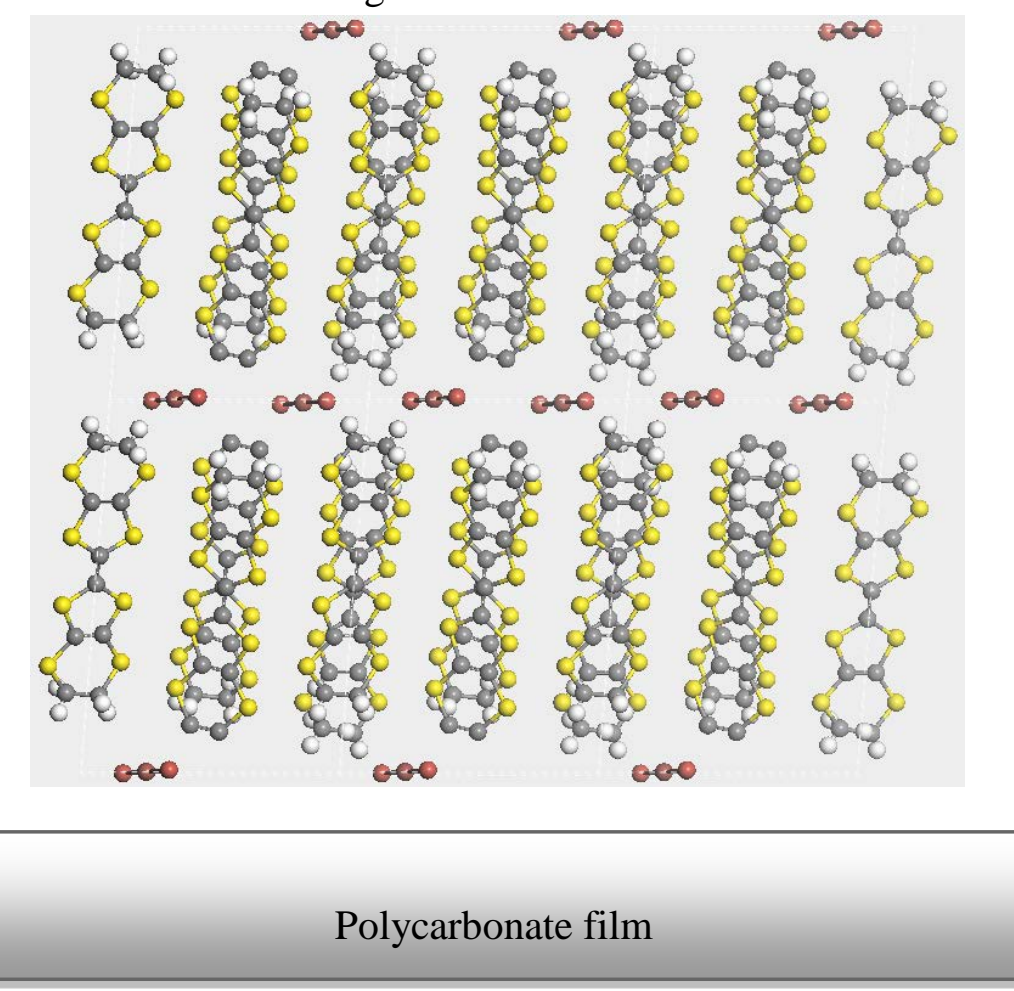

Figure 1. Schematic presentation of the highly temperature sensitive bi-layer film: polycarbonate $/ \alpha^{\prime}$-(BEDT-TTF $)_{2} \mathrm{I}_{\mathrm{x}} \mathrm{Br}_{3-\mathrm{x}}$ showing the (001) orientation of $\alpha^{\prime}$-(BEDT$\mathrm{TTF})_{2} \mathrm{I}_{\mathrm{x}} \mathrm{Br}_{3-\mathrm{x}}$ 
In particular, we present and discuss processing of the thermistor: polycarbonate/ $\alpha^{\prime}-$ $(B E D T-T T F)_{2} \mathrm{I}_{\mathrm{x}} \mathrm{Br}_{3-\mathrm{x}}$ into polyester textile. This process enables engineering of e-textile which can detect even negligible temperature changes. For the sake of feasibility of this research, we implement associated 'proof-of-concept' by designing a wireless sensor node with the proposed thermistor. The resultant monitoring system is capable of precise monitoring of ambient temperature changes in an office environment depending on the presence of working personnel.

\section{Experimental Materials and Methods}

\subsection{Preparation and Characterization of the Flexible Lightweight Bi Layer Thermistor: Polycarbonate/ $\boldsymbol{\alpha}^{\prime}-(\mathbf{B E D T - T T F})_{2} \mathbf{I}_{\mathbf{x}} \mathbf{B r}_{3-\mathrm{x}}$}

For fabricating the BL thermistor we followed the synthetic procedure described in [29]. To be more specific, we first prepared a $25 \mu \mathrm{m}$ thick polycarbonate (PC) film which contains an 8 wt. \% of BEDT-TTF. To effectuate this task, the film is cast on a glass support at $130{ }^{\circ} \mathrm{C}$ from a 1,2-dichlorobenzene solution of polycarbonate and BEDT-TTF. Afterwards, to perform covering of the film with the (001) oriented layer of $\alpha$-(BEDT-TTF $)_{2} \mathrm{I}_{\mathrm{x}} \mathrm{Br}_{3-\mathrm{x}}$, we exposed the film surface to the vapors of a $0.5 \mathrm{M}$ solution of $\mathrm{IBr}$ in dichloromethane for 3 minutes at $30{ }^{\circ} \mathrm{C}$ and relative humidity $40 \%$. For the reasons of temperature and humidity control, we used a climatic chamber MEMMERT HPP. A binary mixture system: IBr/dichloromethane solution$\mathrm{IBr}$ /dichloromethane vapor was used at equilibrium. The X-ray analysis of the resulting surface-modified film indicates the presence of only (00l) reflections being characteristic of conducting layers formed by oriented crystallites (see supplementary material: Fig. S1, ESI). The measured values of the interplanar spacing $d_{001}(16.355 \AA)$ along with intensities of (00l) reflections confirm the formation of the (001) oriented layer of $\alpha^{\prime}$-(BEDT-TTF) $)_{2} \mathrm{I}_{\mathrm{x}} \mathrm{Br}_{3-\mathrm{x}}$, were $0.66>\mathrm{x}>0.33$ [29]. These $\alpha$-(BEDTTTF $)_{2} \mathrm{I}_{\mathrm{x}} \mathrm{Br}_{3-\mathrm{x}}$ linked crystallites contain set of the $\mathrm{IBr}_{2}^{-}, \mathrm{I}_{2} \mathrm{Br}^{-}$, and $\mathrm{I}_{3}{ }^{-}$trihalide anions in which $\mathrm{IBr}_{2}{ }^{-}$anion is a main component [29].

\subsection{Integration of the bi layer thermistor into Polyester Textile}

For forming the rigid support for the bi-layer thermistor, the part of the textile is sandwiched between two glass slides and heated up to $250{ }^{\circ} \mathrm{C}$ (the textile melting point) using soldering iron; the plastification process of polyester fabric is visually controlled. This procedure results in the formation of a smooth rigid polyester film in a heated part of textile. The polycarbonate-based layer of the thermistor: polycarbonate $/ \alpha^{\prime}$-(BEDT-TTF $)_{2} \mathrm{I}_{\mathrm{x}} \mathrm{Br}_{3-\mathrm{x}}$ is attached to the rigid polyester-based support using the glue. This glue is used as it is unable to destroy the polycarbonate layer of the polycabonate/ $\alpha$ '-(BEDT-TTF) $)_{2} \mathrm{I}_{\mathrm{x}} \mathrm{Br}_{3-\mathrm{x}}$ film. To make electrical contacts, four Pt-based 
wirers are attached to the conductive temperature sensing layer of the thermistor using graphite paste. For details see supplementary material Scheme S1, ESI.

\section{Results}

This section shows how the developed bi-layer thermistor: polycarbonate $/ \alpha$ '-(BEDT$\mathrm{TTF})_{2} \mathrm{I}_{\mathrm{x}} \mathrm{Br}_{3-\mathrm{x}}$ can be processed into polyester textile to make a temperature sensing fabric. Fabric-based sensing is a large field of research in the biomedicine. For instance, temperature sensing textile is a promising technology for detecting early signs of breast cancer [30, 31]. The Circadian Biometric Recorder sensors integrated in the bra pick up the minute fluctuations of circadian rhythm-based temperature variances of cell cycles on a 2 to 12 hour bases to identify any abnormality at an early stage of cell augmentation. [30, 31] E-textile can be used in many state-of-the-art sport, military and aerospace technologies as they can guarantee unobtrusive sensing.

\subsection{Embedding of the bi layer thermister: polycarbonate/ $\alpha^{\prime}-(B E D T-T T F)_{2} I_{x} B r_{3-x}$ into polyester textile}

For developing temperature sensing e-fabric using the $\alpha^{\prime}$-(BEDT-TTF) $)_{2} \mathrm{I}_{\mathrm{x}} \mathrm{Br}_{3-\mathrm{x}}$-based conducting layer as an active component one has consider the high sensitivity of the electrical resitance of $\alpha$-(BEDT-TTF $)_{2} \mathrm{I}_{\mathrm{x}} \mathrm{Br}_{3-\mathrm{x}}$ to temperature changes happens along with high sensitivity to deformation [32]. Fig. 2 presents the thermistor polycarbonate $/ \alpha^{\prime}$-(BEDT-TTF $)_{2} \mathrm{I}_{\mathrm{x}} \mathrm{Br}_{3-\mathrm{x}}$ which shows a reversible electrical response to cyclic monoaxial deformation with gage factor $(G F) \cong 8$; $G F=\varepsilon\left(R_{0} / R_{\varepsilon}-R_{0}\right)$, were $R_{0}=$ initial resistance, $\mathrm{R}_{\varepsilon}=$ resistance at relative strain $\varepsilon$.

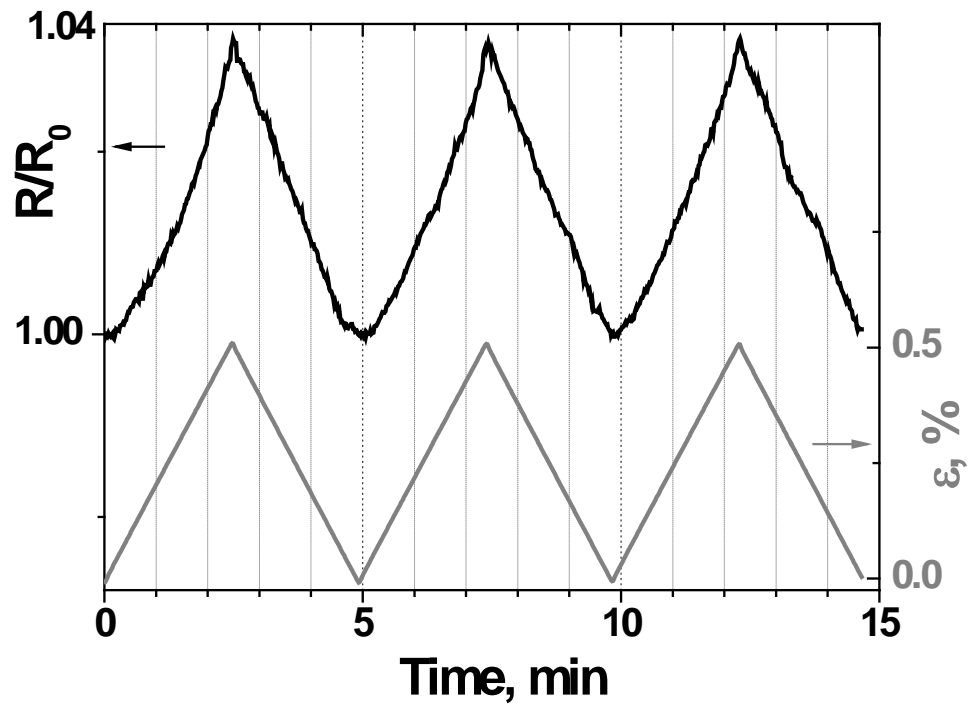

Figure 2. Electrical resistance response of the $\alpha^{\prime}$-(BEDT-TTF $)_{2} \mathrm{I}_{\mathrm{x}} \mathrm{Br}_{3-\mathrm{x}}$ conducting layer to cyclic monoaxial elongations

Hence, when developing the temperature sensing e-textiles at its first phase has to be reduced to engineering a rigid textile-based support to which the thermistor can be 
connected at the second phase. Our material engineering principle is based on Hooke's law. The combination of soft fabric with a rigid textile-based unit may be considered as a combination of two springs in which one (texile) has the spring constant $\left(\mathrm{k}_{1}\right)$ being significantly less comparing to another spring $\left(\mathrm{k}_{2}\right)$ (rigid textile-based unit). Relying on Hooke's law, the extending $(\Delta x)$ of this combination under force $(F)$ is as follows:

$$
\Delta \mathrm{x}=\Delta \mathrm{x}_{1}+\Delta \mathrm{x}_{2}=\mathrm{F} / \mathrm{k}_{1}+\mathrm{F} / \mathrm{k}_{2}
$$

when $\mathrm{k}_{1}<<\mathrm{k}_{2}, \Delta \mathrm{x}_{1}>>\Delta \mathrm{x}_{2}$, the extending of a rigid textile-based unit will be extremely small and therefore the deformation effect will be reduced to a minimum. To form a textile-based rigid unit we have used an impregnation process: a part of a textile is impregnated by rigid polymer (polycarbonate) and then the bi layer thermistor: polycarbonate $/ \alpha^{\prime}$-(BEDT-TTF $)_{2} \mathrm{I}_{\mathrm{x}} \mathrm{Br}_{3-\mathrm{x}}$ is connected to the impregnated part (see supplementary material: Fig. S1, ESI) [30].

At the same time, our recent microscopic study revealed that this 'attaching' approach resulted in the formation of some cracks on the conductive sensing layer of the bi layer thermistor (Fig. 3). This result motivated us to look for another approach for processing the bi layer thermistor into polyester textiles.
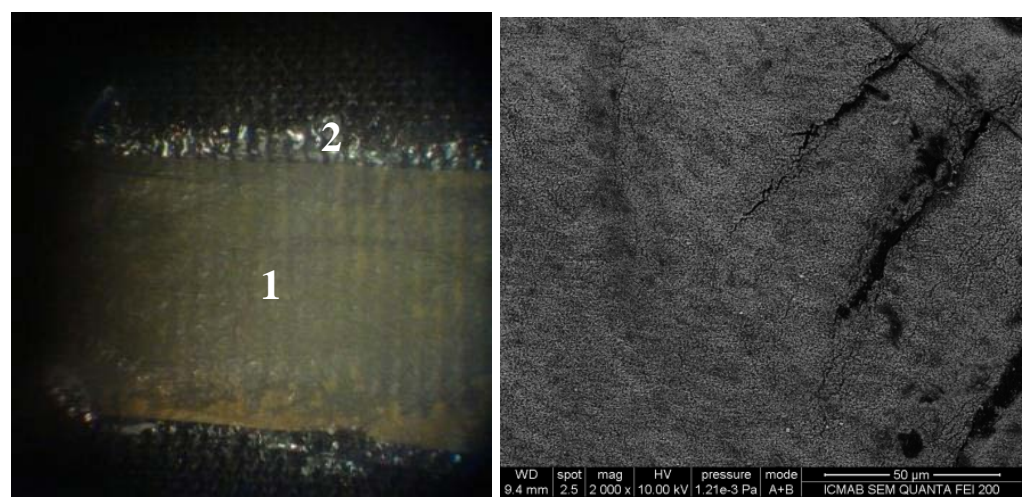

Figure 3. Left: photo image of the one of the parts of the bi layer thermistor (1) that was attached to the rigid impregnated part of the polyester fabric (2); the SEM image of the thermistor showing cracks developed on the conductive sensing layer of the BL thermistor being attached to the polyester fabric

To highlight the formation of the above described crack-like defects, we suggest that a rigid flat unit at the polyester textile to which the thermistor has to be attached may be prepared by locally melting of a small part of the textile being sandwiched between two glass slides. Under local melting the small part of the polyester textile (see supplementary material: ESI, Scheme S2, first phase), has to lose its textile-like texture while becoming film-like. The melted part must become much more rigid as compared to the rest of the textile. The above procedure allowed us to prepare the smoother rigid support as compared to that fabricating by textile impregnation. The bi-layer thermistor is attached to the rigid polyester-based support using the glue (ESI scheme S2, second phase). At the final stage of the prototype fabrication, we attach the electrical contacts to 
the conductive temperature sensing layer of the thermistor using graphite paste (Fig. 4). The SEM image of the surface of the $\alpha^{\prime}$-(BEDT-TTF $)_{2} \mathrm{I}_{\mathrm{x}} \mathrm{Br}_{3-\mathrm{x}}$-based temperature sensing layer shows that it has no imperfectness which can be found in Fig. 3.
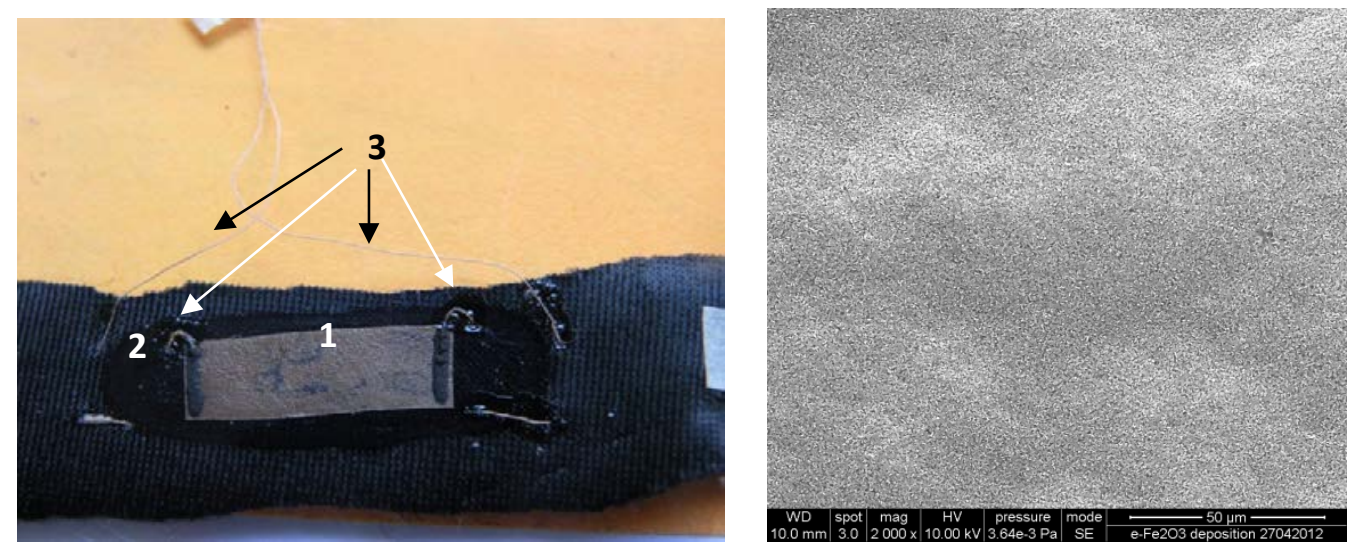

Figure 4. Left: photo image of the developed prototype of temperature sensing e-textile (1-thermistor, 2-rigid film-like unit prepared by local melting of polyester-based textile, 3 electrical connections); right: SEM image of the thermistor showing a very good quality of its conducting layer

\subsection{Temperature testing of the developed prototype e-textile}

We design testbed for validating and experimenting on sensing human body temperature changes. Fig. 5 shows that the resistance of the e-textile strongly depends on temperature: the resistance decreases linearly from 34 to $28.5 \mathrm{k} \Omega$ when temperature increases from 30 to $43{ }^{\circ} \mathrm{C}$. From these data the electrical response of the fabricated etextile to temperature changes equally $\cong 0.42 \mathrm{k} \Omega /$ degree which is a good result in terms of resistance change per degree: $2.1 \Omega$ per 0,005 temperature degree change. The temperature coefficient of resistance is calculated as the relative change of resistance per degree of temperature change, i.e. $\cong-1.2 \%$ /degree that well corresponds to early reported data [33].

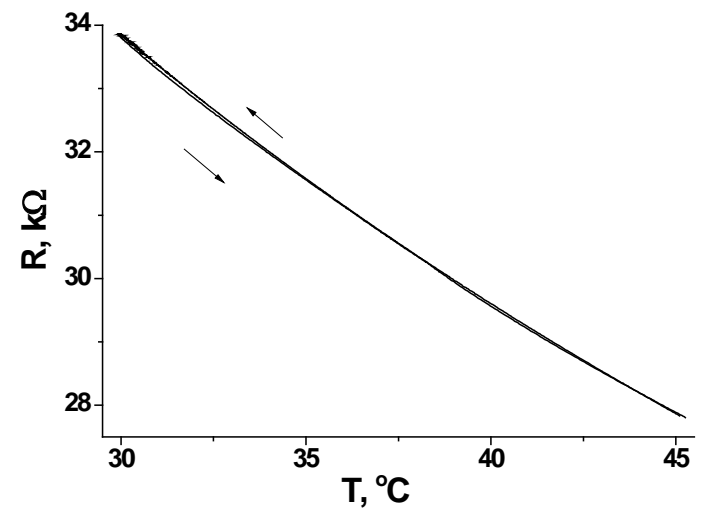


Figure 5. Resistance temperature of the developed e-textile in the body temperature range

We then test the sensing e-fabric at heating cycles from room temperature up to 60 ${ }^{\circ} \mathrm{C}$. This temperature test (Fig. 6) showed that the electrical response of the prototype to temperature cycling is reversible, repeatable and stable in time.

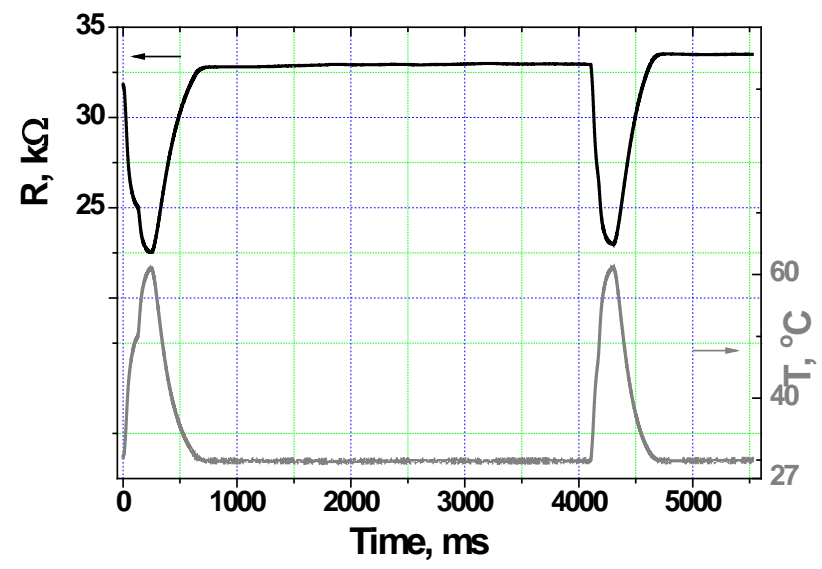

Figure 6. Response of the electrical resistance of the developed e-textile to temperature cycles.

These experimental results ensure that the developed all-organic bi-layer thermistor polycabonate/ $\alpha$-(BEDT-TTF $)_{2} \mathrm{I}_{\mathrm{x}} \mathrm{Br}_{3-\mathrm{x}}$ is promising for application in smart wearable fabrics. In particular, it guarantees the measurement of small changes in a body temperature fluctuations.

\subsection{Interfacing BL-film sensor with wireless sensor node}

In this section we present a custom made wireless sensor node and its interfacing with the BL-film sensor. We deploy this monitoring system in a real facility and conduct temperature measurements.

\subsubsection{Wireless sensor node design}

Fig. 7 presents the architecture of wireless sensor node with the BL-film sensor described in Section 2. The architecture consists of four units: sensing, processing, wireless communication and power supply. The processing unit is built around the ATXmega128 microcontroller unit (MCU) with a precise 24-bit Analog-to-Digital Converter (ADC). Main tasks of MCU include the management of sensor operation and sending measured data to wireless communication unit. It is based on ETRX3 wireless modem able to transmit and receive data from the sensor nodes in the network. Wireless modem has some important self-configuration features which simplify WSN deployment and debugging: WSN configuration, adjustment of TX power. Thermistor 
is connected to the MCU via ADC and is characterized by quick response time and high sensitivity. Power supply unit is comprised of a 3.6 V, 3200 mAh AA Li-Ion cell and power management based on a DC/DC which provides the sensor nodes with a stable 3 V.

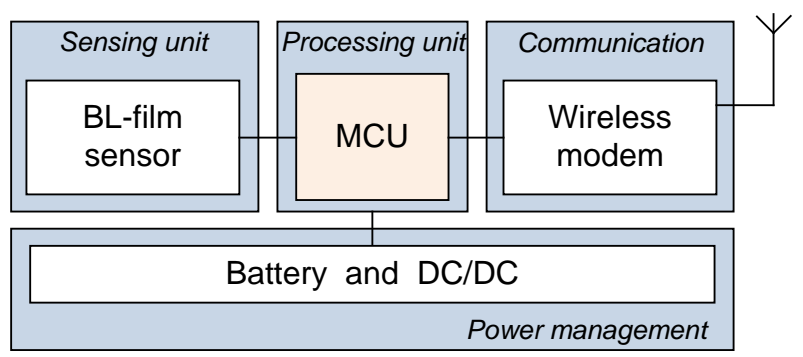

Figure 7. Sensor node architecture.

Fig. 8 shows the prototype of the wireless sensors node equipped with the BL-film sensor. The prototype is a compact sensing device $(8 \times 4.5 \mathrm{~cm})$ with the state-of-the-art sensor and empowered by the wireless communication feature.

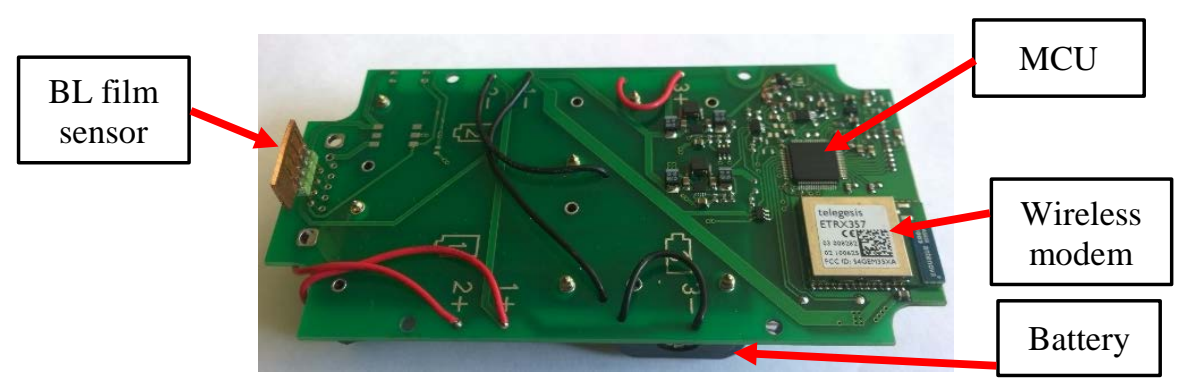

Figure 8. Prototype of the wireless sensor node with the BL-film as a sensing element.

Apart from the sensor which was fabricated from scratch (see Section 2), we used off-the-shelf components for assembling the wireless sensor node. Due to autonomous nature of WSN [12], the key criteria for choosing the electronic components is low power consumption to guarantee the WSN long-term operation. The novelty in the design of the thermistor is the application of "self-metallization" technology. It is helpful for depositing the sensors in the empty spaces on the printed circuit board. We note that for testing reasons we used the thermistor deposited on an extra board.

\subsection{Experimental results}


We assess the prototype of wireless sensor node in a real office environment within one working day. Fig. 9 shows how the resistance of the BL-film changes with respect to the temperature in the office which is served by a Heating, Ventilation and Air Conditioning (HVAC) system. The goal of this experiment is to evaluate the sensitivity of BL-film. Since the temperature in the office can not drastically change and for ensuring energy efficient operation of the node, it set up to conduct the measurement every two minutes and send the data to a network coordinator located in a corridor.

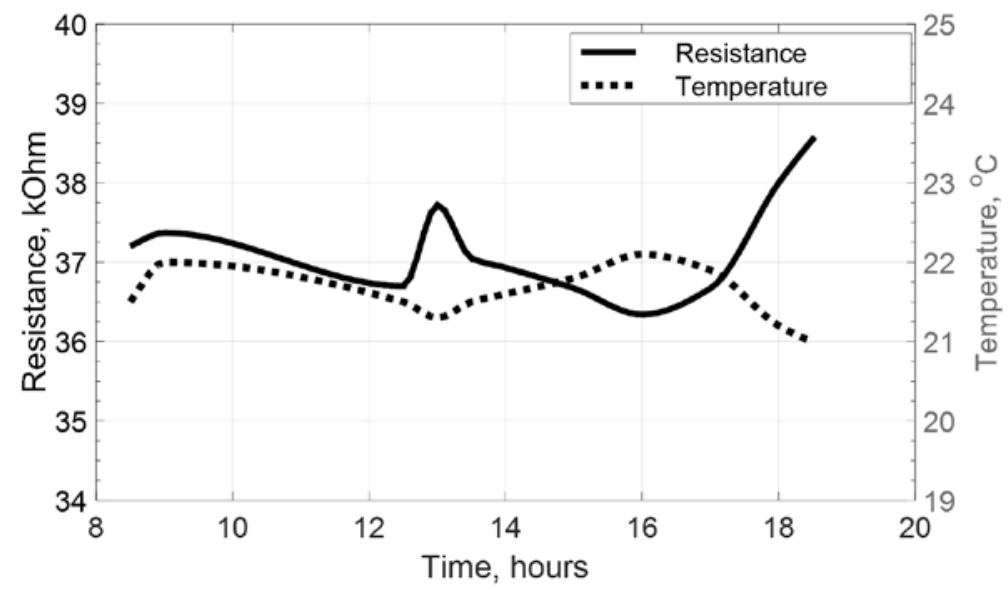

Figure 9. Experiment showing how the film resistance and temperature change in an office environment during the working hours.

The results shown in Fig. 9 demonstrate that the temperature in the office fluctuates in the range $21-22{ }^{\circ} \mathrm{C}$ over time even though it is controlled by the HVAC system. For example, the temperature starts growing in the beginning of working day by 9AM. It happens due to arriving of workers to their office. Then the temperature gradually decreases by 1PM which is most likely associated with the lunch time. Afterwards the temperature starts increasing again when the office workers come back. Finally, the office temperature cools down at the end of working day.

\section{Conclusions}

In this work we have presented the $\mathrm{BL}$ thermistor: polycarbonate/ $\alpha$-(BEDT$\mathrm{TTF})_{2} \mathrm{I}_{\mathrm{x}} \mathrm{Br}_{3-\mathrm{x}}$ and investigated its conductive sensing layer texture and structure by using SEM and X-ray analysis. Also, we measured the resistance temperature dependence of the thermistor. We have demonstrated that all investigated properties are in agreement with earlier reported results and improve them.

Apart from reporting on the BL thermistor we have presented a new approach to integrating the $\mathrm{BL}$ thermistor into textile where the thermistor is attached to the smooth film-like rigid support prepared directly at the polyester textile by its local melting. We discovered that melted part of the textile is much more rigid comparing to the rest of the fabric. 
The fabricated prototype can be used as a temperature sensor for detecting the temperatures in the range from RT to $60{ }^{\circ} \mathrm{C}$. According to the experimental results the electrical response to temperature change is reversible, repeatable and stable in time. Developed textiles can detect even negligible temperature change with accuracy of $0.005{ }^{\circ} \mathrm{C}$. This results improves one obtained for widely used used thermistors where accuracy of a Pt-1000 detector is $0.01{ }^{\circ} \mathrm{C}$.

Finally, we have demonstrated that proposed solution has a number of applications in biomedical and environmental monitoring scenarios by integrating the thermistor with the wireless sensor node for enabling timely delivery of measured phenomena to the user.

\section{Acknowledgements}

The authors acknowledge the financial support from Instituto de Salud Carlos III, through “Acciones CIBER.” The Networking Research Center on Bioengineering, Biomaterials and Nanomedicine (CIBER-BBN), aninitiative funded by the VI National R\&D\&I Plan 2008-2011, Iniciativa Ingenio 2010, Consolider Program, CIBER Actions and financed by the Instituto de Salud Carlos III with assistance from the European Regional Development Fund. The authors also appreciate the financial support through the projects: BE-WELL (CTQ2013-40480-R) granted by DGI (Spain), and GenCat (2014-SGR-17) financed by DGR (Catalunya). The authors further acknowledge financial support from the European Commission's Seventh Framework Programme for Research under contract FP7-OCEAN-2013-614155 and support by the grant No. RFMEFI57714X0133 from the Ministry of Education and Science of Russian Federation.

\section{References}

[1] S.R. Forest, M. E. Thompson, Introduction: Organic Electronics and Optoelectronics, Chemical Reviews. 107 (2007) 923-925.

[2] Organic Electronics for a Better Tomorrow: Innovation, Accessibility, Sustainability, 2011, http://www.rsc.org/globalassets/04-campaigning-outreach/policy/research-policy/globalchallenges/organic-electronics-for-a-better-tomorrow1.pdf

[3] E. Laukhina, R. Pfattner, L. R. Ferreras, S. Galli, M. Mas-Torrent, N. Masciocchi, V. Laukhin, C. Rovira, J. Veciana, Ultrasensitive Piezoresistive All-Organic Flexible Thin Films, J. Adv. Mater. 22 (2010) 977-981.

[4] R. Pfattner, V. Lebedev, E. Laukhina, S. C. Kumar, A. Esteban-Martin, V. RamaiahBadarla, M. Ebrahim-Zadeh, F. P. García de Arquer, G. Konstantatos, V. Laukhin, C. Rovira, J. Veciana, A Highly Sensitive Pyroresistive All-Organic Infrared Bolometer, J. Advanced Electronic Materials, 1 (2015), online publication. DOI: 10.1002/aelm.201500090. 
[5] R. Kondo, M. Higa, S. Kagoshima, H. Hoshino, T. Mori, H. Mori, Electrical and structural properties of thetha-type BEDT-TTF organic conductors under uniaxial strain, J. Phys. Soc. Jpn. 75 (2006) 44716-44717.

[6] V. Laukhin, I. Sánchez, A. Moya, E. Laukhina, R. Martinc, F. Ussc, C. Rovira, A. Guimera, R. Villa, J. Aguiló, J.-C. Pastor, J. Veciana, Non-invasive intraocular pressure monitoring with a contact lens engineered with a nanostructured polymeric sensing film, Sensors and Actuators A, 170 (2011) 36-43.

[7] E. Laukhina, J. Ulanski, A. Khomenko, S. Pesostskii, V. Tkachev, L. Atovmyan, E. Yagubskii, C. Rovira, J. Veciana, J. Vidal-Gancedo, V. Laukhin, Systematic Study of the (ET)2I3 reticulate doped polycarbonate films: structure, ESR, transport properties and superconductivity, J. Phys. I France. 7 (1997) 1665-1675.

[8] D. Miorandi, S. Sicari, F. De Pellegrini, I. Chlamtac, Internet of Things: Vision, Applications and Research Challenges, J. Ad Hoc Networks. 10 (2012) 1497-1516.

[9] D. Kelaidonis, A. Somov, V. Foteinos, G. Poulios, V. Stavroulaki, P. Vlacheas, P. Demestichas, A. Baranov, A.R. Biswas, R. Giaffreda, Virtualization and Cognitive Management of Real World Objects in the Internet of Things. In: IEEE International Conference on Green Computing and Communications (GreenCom), pp.187-194, 2012.

[10] A. Somov, C. C. Ho, R. Passerone, J.W. Evans, P.K. Wright, Towards Extending Sensor Node Lifetime with Printed Supercapacitors. In: Picco, G. P., Heinzelman, W. (eds.) EWSN 2012. LNCS, vol. 7158, pp. 212-227. Springer-Verlag, Berlin, Heidelberg, 2012.

[11] J. Segura-Garcia, S. Felici-Castell, J.J. Perez-Solano, M. Cobos, J.M. Navarro, Low-Cost Alternatives for Urban Noise Nuisance Monitoring Using Wireless Sensor Networks, J. IEEE Sensors Journal. 15 (2015) 836-844.

[12] A. Somov, A. Baranov, D. Spirjakin, R. Passerone, Circuit Design and Power Consumption Analysis of Wireless Gas Sensor Nodes: One-Sensor Versus Two-Sensor Approach, J. IEEE Sensors. 14 (2014) 2056-2063.

[13] T. Dutta, Medical Data Compression and Transmission in Wireless Ad Hoc Networks, J. IEEE Sensors. 15 (2015) 778-786.

[14] A. Zanella, N. Bui, A. Castellani, L. Vangelista and M. Zorzi, Internet of Things for Smart Cities, J. IEEE Internet of Things. 1 (2014) 22-32. doi: 10.1109/JIOT.2014.2306328.

[15] L. Y. Mano, B. S. Faiçal, L. H.V. Nakamura, P. H. Gomes, G. L. Libralon, R. I. Meneguete, G. P.R. Filho, G. T. Giancristofaro, G. Pessin, B. Krishnamachari, J. Ueyama, Exploiting IoT technologies for enhancing Health Smart Homes through patient identification and emotion recognition, J. Computer Communications. 89-90 (2016) 178-190. Doi:10.1016/j.comcom.2016.03.010.

[16] A. C. Sun, C. Yao, A.G. Venkatesh, D. A. Hall, An efficient power harvesting mobile phone-based electrochemical biosensor for point-of-care health monitoring, Sensors and Actuators B: Chemical, 235 (2016) 126-135. Doi: 10.1016/j.snb.2016.05.010.

[17] S. Khan, L. Lorenzelli, R. S. Dahiya, Technologies for Printing Sensors and Electronics Over Large Flexible Substrates: A Review, J. IEEE Sensors. 15 (2015) 3164-3185. 
[18] D. De Rossi, F. Carpi, F. Lorussi, A. Mazzoldi, R. Paradiso, E. P. Scilingo, A. Tognetti, Electroactive Fabrics and Wearable Biomonitoring Devices, AUTEX Research Journal, 3 (2003) 180-185.

[19] K.L. Mittal: Preface in Metallized Plastics 7: Fundamental and Applied Aspects, Ed. K.L. Mittal, VSP BV, vii (2001).

[20] C-C. Huang, Zh-K. Kao, Y-Ch.Liao, Flexible Miniaturized Nickel Oxide Thermistor Arrays via Inkjet Printing Technology, ACS Appl. Mater. Interfaces. 5 (2013) 12954-12959.

[21] R. J. Waltman, J.Bargon, Electrically conducting polymers: a review of the electropolymerization reaction, of the effects of chemical structure on polymer film properties, and of applications towards technology, Canadian Journal of Chemistry. 64 (1986) 76-95.

[22] S. Pimanpang, P.I. Wang, G.C. Wang, T.M. Lu, Self-assembled monolayer growth on chemically modified polymer surfaces, Applied Surface Science. 253 (2006) 3532-3540.

[23] S. Joo, D. F. Baldwin, Adhesion mechanisms of nanoparticle silver to substrate materials: identification, J. Nanotechnology. 21 (2010), electronic publication 055204. doi: 10.1088/09574484/21/5/055204.

[24] E.E. Laukhina, V.A. Merzhanov, S.I. Pesotskii, A.G. Khomenko, E.B. Yagubskii, J. Ulanski, M. Kryszewski, J.K. Jeszka, Superconductivity in reticulate doped polycarbonate films, containing (BEDT-TTF)2I3, J. Synthetic Metals. 70 (1995) 797-800.

[25] E. Laukhina, V. Tkacheva, S. Khasanov, L. Zorina, J. Gomez-Segura, A. Perezdel Pino, J. Veciana, V. Laukhin, C.Rovira, Linked Crystallites in the Conducting Topmost Layer of Polymer Bilayer Films Controlled by Temperature: From Micro- to Nanocrystallites, J. ChemPhysChem. 7 (2006) 920-923.

[26] V. Lebedev, E. Laukhina, C. Rovira, V. Laukhin, J. Veciana, All-Organic Humidity Sensing Films with Electrical Detection Principle Suitable to Biomedical Applications, J. Procedia Engineering. 47 (2012) 603-606.

[27] L. R. Ferreras, R. Pfattner, M. Mas-Torrent, E. Laukhina, L. Lopez, V. Laukhin, C. Rovira, J. Veciana, Highly piezoresistive textiles based on a soft conducting charge transfer salt, J. Mater. Chem. 21 (2011) 637-640.

[28] PT-100 Series Platinum RTDs:

http://www.lakeshore.com/Documents/LSTC_Platinum_l.pdf

[29] E. Laukhina, V. Tkacheva, I. Chuev, E. Yagubskii, J. Vidal-Gancedo, M. Mas-Torrent, C. Rovira, J. Veciana, S. Khasanov, R. Wojciechowski, J. Ulanski, New Flexible Low-Density Metallic Materials Containing the (BEDT-TTF $)_{2}\left(\mathrm{I}_{\mathrm{x}} \mathrm{Br}_{1-\mathrm{x}}\right)_{3}$ Molecular Metals as Active Components, J. Phys. Chem. B. 105 (2001) 105 11089-11097.

[30] J. Hsu, October 17, 2012, http://www.livescience.com/24074-smart-bra-detect-cancer.html [31] Published 06/03/2014 By "Nuviun digital health” 
[32] E. Laukhina, V. Lebedev, C. Rovira, V. Laukhin, J Veciana, Attractive mechanical properties of a lightweight highly sensitive bi layer thermistor: polycarbonate/organic molecular conductor, IOP Conf. Series: Materials Science and Engineering 108 (2016) 012050.

[33] E. Laukhina, V. Laukhin, V. Lebedev, C. Rovira, J. Veciana, Conductive fabric responding to extremely small temperature changes, J. Procedia Engineering. 87 (2014) 144 - 147. 